\title{
A THEOREM CONCERNING SIMPLY TRANSITIVE PRIMITIVE GROUPS*
}

\author{
BY W. A. MANNING
}

The theorem here presented has evolved by easy stages from a paragraph in Jordan's Memoir on primitive groups. $\dagger$ In the discussion of a particular class of simply transitive primitive groups, he showed that the degree of a doubly transitive constituent of the subgroup leaving one letter fixed cannot be greater than the sum of the degrees of all the other transitive constituents.

TheOREm. Let $H$ be the subgroup that fixes one letter of a simply transitive primitive group. If one of the constituents of $H$ is a doubly transitive group of degree $m$, there is in $H$ a transitive constituent whose degree is greater than $m$ and divides $m(m-1)$.

Let $G$, of degree $n$ and of order $n h$, be the given simply transitive primitive group. Let $H$, the subgroup of $G$ that leaves the letter $x$ fixed, be denoted by $G(x)$.

Let it be assumed: (1) that $G(x)$ has exactly $k$ similar doubly transitive constituent groups: $A$ on the letters $a_{1}, a_{2}, \cdots, a_{m} ; B$ on $b_{1}, b_{2}, \cdots, b_{m} ; \cdots ; K$ on $k_{1}, k_{2}, \cdots$, $k_{m}$; (2) that $G\left(a_{1}\right)$ has $k-1$ doubly transitive constituents: $B_{1}$ on $b_{1}, a_{2}, \cdots, a_{m} ; C_{1}$ on $c_{1}, b_{2}, \cdots, b_{m} ; \cdots ; K_{1}$ on $k_{1}$, $j_{2}, \cdots, j_{m}$; (3) that $n$ is greater than $k m+1$. These assumptions, when $k=1$, reduce to the hypothesis of our theorem. We wish to prove by induction that there is a transitive

* Presented to the Society, April 6, 1928.

$\dagger$ C. Jordan, Bulletin de la Société Mathématique de France, vol. 1 (1873), p. 198, §64.

Manning, American Journal of Mathematics, vol. 39 (1917), p. 298; Transactions of this Society, vol. 20 (1919), p. 66; Primitive Groups, 1921, p. 83; Transactions of this Society, vol. 29 (1927), p. 821, §8.

$\ddagger$ Manning, Transactions of this Society, vol. 29 (1927), p. 821, §8. 
constituent of some degree $\mu$ in $G(x)$ such that $\mu$ divides $m(m-1)$ and is greater than $m$.

The letters of $G$ are supposed to be so chosen that each substitution of $G(x)$ permutes the $m$ subscripts of the letters of the similar groups $A, B, \cdots, K$ in exactly the same way.

The subgroup $G(x)\left(a_{1}\right)$ of $G(x)$ (that fixes $a_{1}$ ) is of order $h / m$. The subgroups $G(x)\left(a_{1}\right)$ and $G\left(a_{1}\right)(x)$ are identical, and therefore in $G\left(a_{1}\right) x$ is a letter of a transitive constituent of degree $m . G\left(a_{1}\right)(x)\left(=G\left(b_{1}\right)(x)=G\left(c_{1}\right)(x)=\cdots=G\left(k_{1}\right)(x)\right)$, because of assumption (1) has $k$ transitive constituents of degree $m-1$ each on the letters $a_{2}, a_{3}, \cdots, a_{m} ; b_{2}, b_{3}, \cdots$, $b_{m} ; \cdots ; k_{2}, k_{3}, \cdots, k_{m}$, respectively. Since $G$ is primitive, $\left\{G(x), G\left(a_{1}\right)\right\}=G$, and therefore $G\left(a_{1}\right)$ cannot have a transitive constituent of degree $m-1$ on the letters $k_{2}, k_{3}, \cdots, k_{m}$, nor a transitive constituent of degree $m$ on the letters $x$, $k_{2}, \cdots, k_{m}$. In $G\left(a_{1}\right)$ the letters $k_{2}, k_{3}, \cdots, k_{m}$ all belong to the same transitive constituent of unknown degree $\mu(\geqq m)$. Then $G\left(a_{1}\right)\left(k_{2}\right)$ is of order $h / \mu$ and if $x$ belongs to a transitive constituent of degree $\delta(\geqq 1)$ in $G\left(a_{1}\right)\left(k_{2}\right), G\left(a_{1}\right)\left(k_{2}\right)(x)$ is of order $h / \mu \delta$. Then since $G(x)\left(a_{1}\right)\left(k_{2}\right)=G(x)\left(k_{1}\right)\left(k_{2}\right), h / \mu \delta$ $=h /[m(m-1)]$, and $\mu$ divides $m(m-1)$. Either our theorem is proved or $\mu=m$. If $\mu=m, G\left(a_{1}\right)$ has this transitive constituent $\left(L_{1}\right)$ on the letters $l_{1}, k_{2}, \cdots, k_{m}$ (where $\left.l_{1} \neq x\right) ; L_{1}$ is doubly transitive because it contains a transitive subgroup of degree $m-1$ on $k_{2}, k_{3}, \cdots, k_{m}$.

The substitution $\left(a_{1} a_{i} \cdots\right) \cdots$ of $G(x)$ transforms $G\left(a_{1}\right)$ into $G\left(a_{i}\right)$ and $L_{1}$ into $L_{i}$, a doubly transitive constituent of $G\left(a_{\imath}\right)$ on the $m$ letters $l_{i}, k_{1}, k_{2}, \cdots, k_{i-1}, k_{i+1}, \cdots, k_{m}\left(l_{i} \neq x\right)$. Nor is $l_{i}=l_{1}$, for if it were, $\left\{G\left(a_{1}\right), G\left(a_{i}\right)\right\}$ would have a transitive constituent of degree $k m+1(<n)$ on the letters $a_{1}, a_{2}, \cdots, k_{m}, l_{1}$. Then $l_{1}, l_{2}, \cdots, l_{m}$ are the letters of a transitive constituent $L$ of $G(x)$. Any substitution of $G(x)$ that replaces $a_{1}$ by $a_{i}$ replaces $l_{1}$ by $l_{i}$, so that the substitutions of $G(x)$ permute the letters of $A$ and the letters of $L$ in exactly the same way. The groups $A$ and $L$ are similar.

Suppose now that $n=(k+1) m+1$. Then $G\left(a_{1}\right)$ has a transitive constituent $X_{1}$ on the $m$ letters $x, l_{2}, \cdots, l_{m}$. $G\left(a_{1}\right)(x)$ 
( $\left.=G\left(b_{1}\right)(x)=\cdots=G\left(l_{1}\right)(x)\right)$ has $k+1$ transitive constituents on $a_{2}, a_{3}, \cdots, a_{m} ; b_{2}, b_{3}, \cdots, b_{m} ; \cdots$; and $l_{2}, l_{3}, \cdots, l_{m}$. No two of the groups $G\left(b_{1}\right), G\left(c_{1}\right), \cdots, G\left(l_{1}\right)$ can have the transitive constituent $a_{2}, a_{3}, \cdots$ in common. Because $B_{1}$ (on $b_{1}, a_{2}, \cdots, a_{m}$ ) is in $G\left(a_{1}\right)$, in one of these $k$ groups $x$, $a_{2}, \cdots, a_{m}$ are the letters of a doubly transitive constituent. Let these subgroups be transformed by $(x)\left(a_{1} a_{2} \ldots\right) \cdots$ into $G\left(b_{2}\right), G\left(c_{2}\right), \cdots, G\left(l_{2}\right)$. One of the transformed groups has a doubly transitive constituent on the letters $x, a_{1}, a_{3}, \cdots, a_{m}$, and therefore contains a substitution $S=\left(x a_{1}\right) \cdots$ which permutes the letters $a_{3}, a_{4}, \cdots, a_{m}$ among themselves. Then $S$ should transform the constituent $A$ of $G(x)$ into the constituent $X_{1}$ of $G\left(a_{1}\right)$; but this, because $m \geqq 3, S$ cannot do. Hence $n>(k+1) m+1$. We have shown too that $G(x)$ has $k+1$ doubly transitive constituents $A, B, \cdots, L$ whose letters are permuted by $G(x)$ in exactly the same way, and that $G\left(a_{1}\right)$ has $k$ transitive constituents $B_{1}, C_{1}, \cdots, L_{1}$. Thus the three conditions of our assumption are reproduced with $k+1$ replacing $k$.

Since $n$ is finite, this process can lead only to the conclusion that the degree of some transitive constituent of $G(x)$ is greater than $m$ and divides $m(m-1)$.

STANFORD UNIVERSITY 